\title{
Ethene to Graphene: Surface Catalyzed Chemical Pathways, Intermediates, and Assembly
}

\author{
Bo Wang, ${ }^{\dagger,}, \|$ Michael König, ${ }^{\dagger, \|}$ Catherine J. Bromley, ${ }^{\ddagger}$ Bokwon Yoon, ${ }^{\S}$ Michael-John Treanor, ${ }^{\ddagger}$

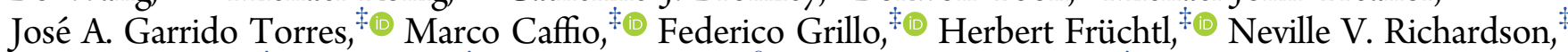

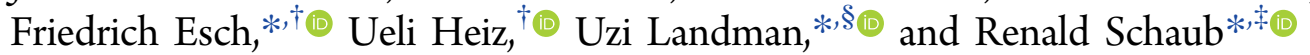 \\ †Chemistry Department, Technische Universität München, 85748 Garching, Germany \\ ${ }^{*}$ EaStCHEM and School of Chemistry, University of St. Andrews, St. Andrews, KY16 9ST, United Kingdom \\ ${ }^{\S}$ School of Physics, Georgia Institute of Technology, Atlanta, Georgia 30332-0430, United States
}

\section{Supporting Information}

\begin{abstract}
Diverse technologies from catalyst coking to graphene synthesis entail hydrocarbon dehydrogenation and condensation reactions on metals and assembly into carbon overlayers. Imperative to gaining control over these processes, through thermal steering of the formation of polyaryl intermediates and the controlled prevention of coking, is the exploration and elucidation of the detailed reaction scheme that starts with adsorbed hydrocarbons and culminates with the formation of extended graphene. Here we use scanning tunneling microscopy, high-resolution electron energy loss and thermal desorption spectroscopies, in combination with theoretical simulations to uncover the hierarchy of pathways

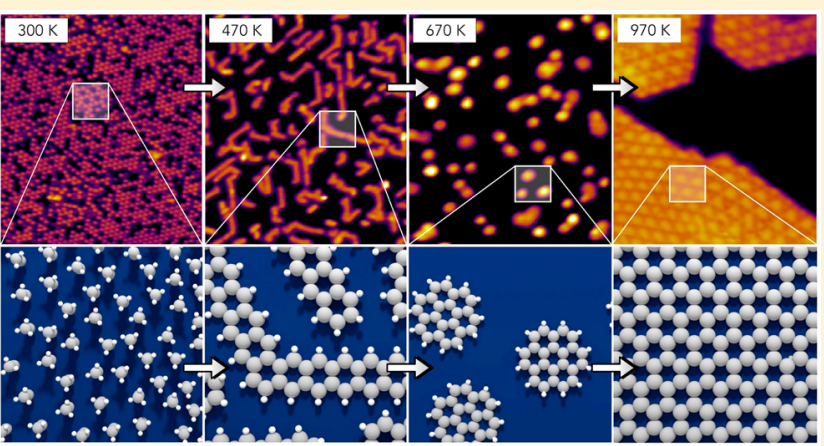
and intermediates underlying the catalyzed evolution of ethene adsorbed on $\mathrm{Rh}(111)$ to form graphene. These investigations allow formulation of a reaction scheme whereby, upon heating, adsorbed ethene evolves via coupling reactions to form segmented one-dimensional polyaromatic hydrocarbons (1D-PAH). Further heating leads to dimensionality crossover $(1 \mathrm{D} \rightarrow 2 \mathrm{D})$ and dynamical restructuring processes at the PAH chain ends with subsequent activated detachment of size-selective carbon clusters. Rate-limiting diffusional coalescence of these dynamically selfevolved precursors culminates $(\leq 1000 \mathrm{~K})$ in condensation into graphene of high structural perfection.
\end{abstract}

\section{INTRODUCTION}

The formation of solid carbonaceous, carbidic, or graphitic material known as coke, ${ }^{1,2}$ derived through anaerobic processing of organic material on metal surfaces at elevated temperatures, has been known since antiquity and patented since the late 16th century for iron ore smelting and making of steel. ${ }^{3}$ Later, it has been found that coking processes, involving catalytic dehydrogenation, polymerization, and cyclization of hydrocarbons, are detrimental to the effective operation of heterogeneous catalytic reactors, necessitating regeneration or exchange of fouled catalytic material. ${ }^{1,2}$ On the other hand, as shown here, somewhat serendipitously, the thermally activated surface-catalyzed reaction processes that underlie undesirable coking may be steered toward the synthesis of a graphene overlayer of macroscopic lateral dimensions, highly valued for its unique physical and chemical properties. ${ }^{4,5}$

Following the isolation of graphene in $2004,{ }^{4}$ top-down methods, ${ }^{6-10}$ including plasma etching lithography and carbon nanotube unzipping, as well as bottom-up techniques, ${ }^{5,11-14}$ based on chemical vapor deposition (CVD) or the application of surface-supported organic synthesis routes (e.g., coupling of polyphenylene precursors on metal substrates) have been employed in preparation of graphene structures with ever increasing perfection (see ref 15 for discussion of recent atomically precise bottom-up preparations). Early investigations yielded a wealth of information about the surface chemistry (e.g., catalyzed $\mathrm{C}-\mathrm{H}$ bond activation and coupling reactions) of small alkane and alkene molecules on metal surfaces (see refs 16 and 17, and references therein). More recent studies (in particular on ethene deposited on transition metal surfaces) have utilized modern surface science techniques (low-energy electron microscopy, low-energy electron microscopy, ${ }^{18,19} \mathrm{X}$ ray photoelectron spectroscopy, ${ }^{20}$ and scanning tunneling microscopy $\left.(\mathrm{STM})^{21}\right)$, extended observations into the graphene formation domain, and resulted in valuable insights into the growth kinetics, suggesting the role of carbon clusters (comprised of $5,^{18,19} 21,22,23$ or $24^{21}$ atoms) whose attachment, rather than that of monomers, leads to ripening and graphene growth; the carbon-cluster hypothesis has been incorporated in a phenomenological kinetic Monte Carlo simulation of the growth process. $^{24}$

Received: March 1, 2017

Published: March 14, 2017 


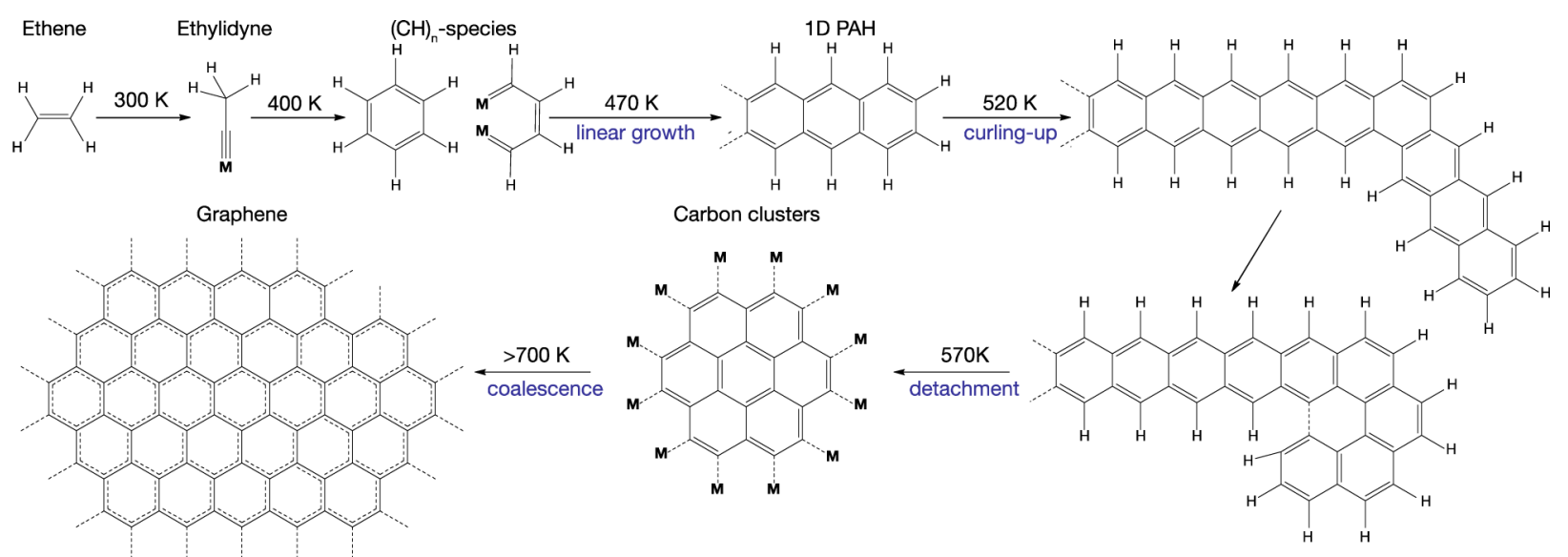

Figure 1. Schematic of the pathway describing the evolution of adsorbed ethene (top left) to graphene (bottom left). The sequence of intermediates identified in this study and their respective appearance temperatures are indicated. At the final stages, following heating to $T>570 \mathrm{~K}$ (see bottom row), coronene-like clusters form. The diffusional coalescence of these precursors results in graphene growth at $T>700 \mathrm{~K}$.

In the next section, we present our results and discussion. We begin with an overview of the reaction scheme starting from ethene and ending with graphene and then discuss in detail experimental and theoretical results pertaining to the different steps in the general reaction scheme. These include ethene deposition and early reaction stages; formation of segmented one-dimensional polyaromatic hydrocarbon (1D-PAH) narrow ribbons; kinking and one-dimensional to two-dimensional (1D $\rightarrow$ 2D) dimensionality crossover of the 1D-PAH chains; theoretically simulated pathways and microscopic formation mechanisms of the self-evolved 24-carbon-cluster precursors and graphene growth. After the concluding remarks, we describe the experimental methods, and details of the computational techniques used in our investigations.

\section{RESULTS AND DISCUSSION}

Reaction Scheme. The research reported herein focuses on gaining insights into the atomic-scale mechanisms of bottom-up chemical pathways that govern the molecular assembly processes resulting in formation of extended graphene structures. Whereas most bottom-up processes start from already rather well-developed polyaryl or oligophenylene precursors (via ex situ synthetic chemistry), we uncover the in situ self-formation of the precursors as integral part of the reaction scheme, starting from the decomposition of a simple hydrocarbon. A scheme derived from our joint experimental and theoretical investigations of the surface-catalyzed thermally activated reaction that transforms ethene adsorbed on $\mathrm{Rh}(111)$ into graphene is shown in Figure 1. This chemical transformation results from annealing the initial $\mathrm{C}_{2} \mathrm{H}_{4} / \mathrm{Rh}(111)$ system at successively higher temperatures; alternatively, it evolves in the course of in situ experiments conducted on a metal surface dosed with ethene at elevated temperatures that match the annealing temperatures used. The scheme shows the key reaction intermediates and their appearance temperatures for the entire ethene to graphene transformation with the corresponding experimental and theoretical findings discussed and illustrated in Figures 2, 3, and 4 below, as well as in the Supporting Information (SI). The reaction scheme presented in this paper has been validated for varying initial ethene exposures up to 1 monolayer. Herein, we present the results pertaining to an initial feedstock exposure of near saturation coverage.
For ease of navigation, we overview here the reaction steps and intermediates/precursors revealed in this study: (i) Adsorption of ethene and its subsequent partial dehydrogenation yielding ethylidyne ( $\equiv \mathrm{C}-\mathrm{CH}_{3} ;$ Figure $2,300 \mathrm{~K}$ ), resulting in the formation of a $\mathrm{C}_{4}$ metallocycle coordinated to surface metal atoms (but-2-en-1,4-diylidene, $\mathrm{M}>\mathrm{C}-\mathrm{C}=\mathrm{C}-$ $\mathrm{C}<\mathrm{M}$; Figure 2, $370 \mathrm{~K}$ ). (ii) Formation of benzene-like $\mathrm{C}_{6}$ intermediates (Figure 2, $370 \mathrm{~K}$ ) and subsequent growth, through successive $\mathrm{C}_{4}$ additions via surface-assisted DielsAlder condensation, of kinked linear 1D-PAH chains, consisting of $\leq 3 \mathrm{~nm}$ long 12-benzene-ring segments with adjacent segments joined by a $120^{\circ}$ kink with respect to each other (Figure 1, rightmost configuration in the top row; Figure 2, $470 \mathrm{~K})$. (iii) Chain-end restructuring with subsequent detachment and dehydrogenation of a coronene-like terminal unit (Figure 1, rightmost configuration in the bottom row; Figure 2, $570 \mathrm{~K}$; see microscopic detachment mechanism in Figure 4) that results in the emergence of a unique size-selective carbon species (Figure 1, middle of bottom row; Figure 2, $670 \mathrm{~K}$ )), identified as the key self-evolved precursor, whose diffusional assembly culminates in the growth of an extended graphene adlayer (Figure 2, 770-970 K). To complement Figure 2b, a collection of STM images acquired at various magnifications for all annealing stages described above is reported in SI4 with the view to provide in-depth insights on the various temperaturedependent intermediates.

Activating and unveiling the above sequence of reaction steps was made possible through the implementation of a carefully programmed sequence of temperature windows, which enabled access to the individual reaction stages, one at a time, thus deconvoluting the complex reaction scheme. In contrast, when dosing the $\mathrm{Rh}(111)$ surface with ethene immediately at high temperatures (e.g., $T \geq 520 \mathrm{~K}$ ), a number of dehydrogenation steps are activated simultaneously and as a result a thin amorphous carbon film forms; see a similar result for a $\mathrm{Ru}(0001)$ surface in ref 25 .

Ethene Deposition and Early Reaction Stages. The widespread of activation energies associated with the catalytic dehydrogenation processes occurring at different stages of the ethene to graphene transformation is reflected in the broad temperature range of the hydrogen Thermal Desorption Spectroscopy (TDS), measured after saturating the surface with ethene at $330 \mathrm{~K}$ (Figure 2a). The first TDS regime, 

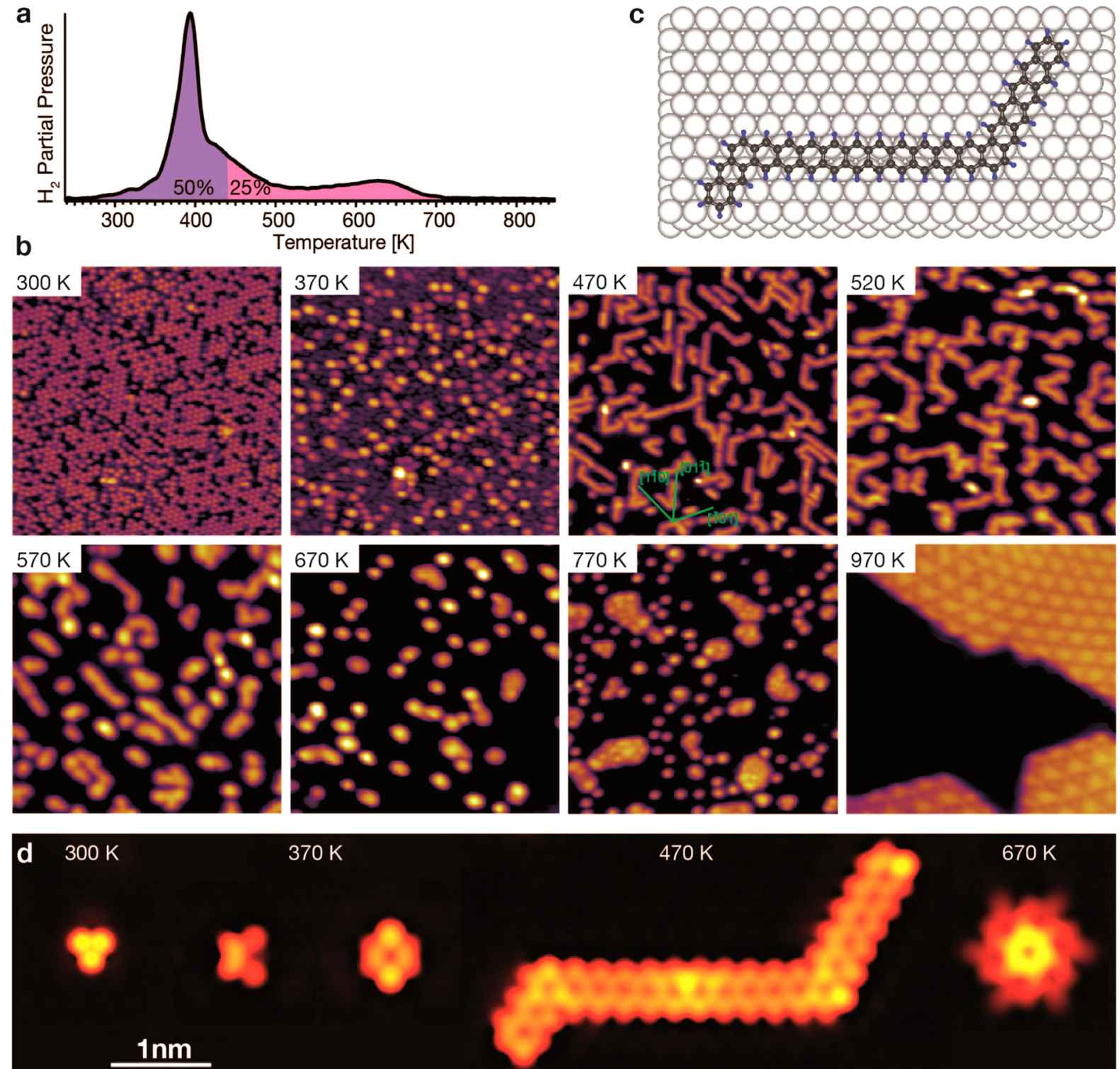

$370 \mathrm{~K}$
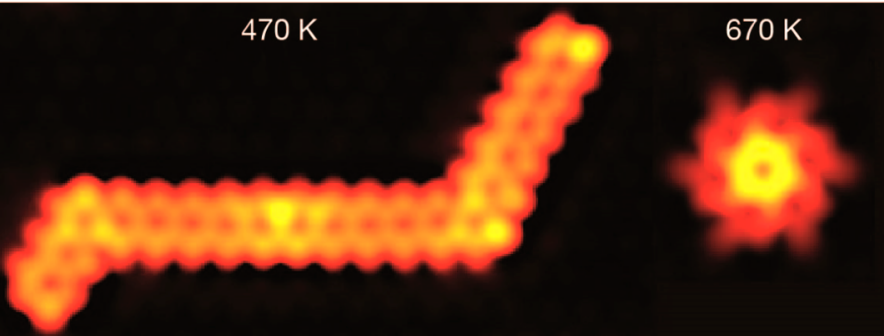

Figure 2. Measured (SI4, SI5) and theoretically simulated (SI7) STM images of stages in the dehydrogenation process in annealing experiments from ethene to size-selective carbon precursors and graphene. (a) TDS of $\mathrm{H}_{2}$ from a $\mathrm{Rh}(111)$ surface saturated with ethene at $330 \mathrm{~K}$. (b) Corresponding low-temperature STM images of the resulting surface species after stepwise flash-annealing to $300 \mathrm{~K},(2 \times 2)$ superstructure of ethylidyne seen as $80 \mathrm{pm}$ protrusions (see also HREELS data in agreement with earlier studies, SI3) (0.98 V; $0.12 \mathrm{nA})$. At $370 \mathrm{~K}$, disordered species, consisting of $\mathrm{H}$ atoms (apparent height, $40 \mathrm{pm})$ and covalently linked intermediates: $\mathrm{C}_{4}(140 \mathrm{pm})$ and $\mathrm{C}_{6}(100 \mathrm{pm}$, see SI5) $(0.11 \mathrm{~V} ; 0.11 \mathrm{nA})$. At $470 \mathrm{~K}, 1 \mathrm{D}-\mathrm{PAHs}$ of various lengths, made of 12-benzene-ring linear segments $(1.11 \mathrm{~V} ; 0.11 \mathrm{nA})$. At $520 \mathrm{~K}$, 1D-PAHs with increased number of kinks, leading to an apparent broadening and reduced length $(1.07 \mathrm{~V} ; 0.11 \mathrm{nA})$. At $570 \mathrm{~K}$, 1D-PAHs shortened by detachment of dome-shaped coronene-type clusters $(0.60 \mathrm{~V} ; 0.12 \mathrm{nA})$. At $670 \mathrm{~K}$, almost complete conversion of the PAH chains into size-selective carbon precursors that diffuse and coalesce into graphene $(0.45 \mathrm{~V} ; 0.12 \mathrm{nA})$. At $770 \mathrm{~K}$, bimodal distribution of carbon precursors and small, defect-rich graphene flakes (1.00 V; $0.11 \mathrm{nA})$. At $970 \mathrm{~K}$, extended graphene islands of high structural quality $(1.00 \mathrm{~V} ; 0.11 \mathrm{nA})$. STM images from 300 to $670 \mathrm{~K}$ are $22 \times 22 \mathrm{~nm}^{2}$ and are displayed with the same topographic contrast (identical $z$-scale). STM images for 770 and $970 \mathrm{~K}$ are $35 \times 35 \mathrm{~nm}^{2}$ and are displayed with the same topographic contrast (but different to previous ones). (c) The relaxed structure of a doubly kinked 19 benzene-ring long 1D-PAH, obtained from first-principle simulations, underlying the simulated STM micrograph shown in (d) for the $470 \mathrm{~K}$ panel in (d). (d) Simulated STM images of the relevant intermediates at each temperature based on the DFT-optimized geometries presented in SI7: $\mathrm{C}_{2} \mathrm{H}_{3}$ at $300 \mathrm{~K}, \mathrm{C}_{4}$ and $\mathrm{C}_{6}$ species at $370 \mathrm{~K}$, $1 \mathrm{D}-\mathrm{PAH}$ at $470 \mathrm{~K}$, and coronene-like precursors at $670 \mathrm{~K}$.

occurring for $T \leq 450 \mathrm{~K}$, consists mainly of a prominent peak at $T \approx 400 \mathrm{~K}$, whereas the second one, at $450 \mathrm{~K} \leq T \leq 700 \mathrm{~K}$, is rather featureless. Early studies ${ }^{26}$ revealed that ethene adsorption at $300 \mathrm{~K}$ on $\mathrm{Rh}(111)$ proceeds initially via the formation of ethylidyne, accounting for $25 \%$ hydrogen loss (one out of every four $\mathrm{H}$ atoms of ethene). The TDS in Figure 2a hence monitors the loss of the three remaining $\mathrm{H}$ atoms in proportions of $50 \%$ and $25 \%$ for the low- and highertemperature regimes, respectively.

Following deposition of ethene on $\mathrm{Rh}(111)$ at room temperature, the ethylidyne species are readily observable in our STM images as protrusions with an apparent height of $80 \mathrm{pm}$ (Figure $2 \mathrm{~b}$ ). As expected, these form an ordered $(2 \times 2)$ superstructure. ${ }^{16,27,28}$ Our corresponding HREELS data shown in Figure 3 (details in SI3) is in good agreement with that 

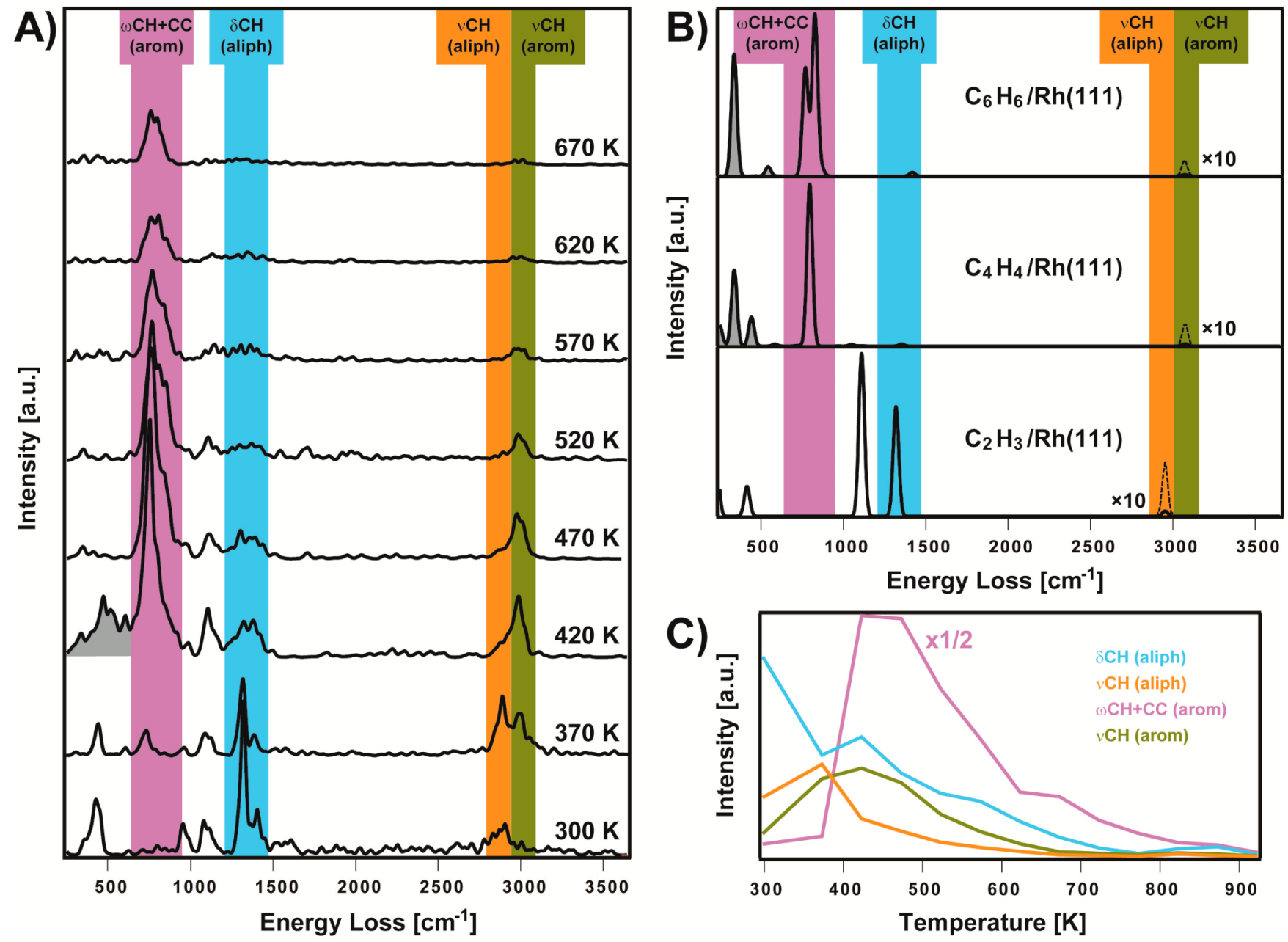

Figure 3. Experimental and DFT-computed HREEL spectra. (A) Experimental HREEL spectra acquired after successive annealings of the ethene/ $\mathrm{Rh}(111)$ system. A detailed discussion and interpretation are reported in SI5. The colored bars highlight vibrational fingerprints of the system: purple, $\mathrm{C}-\mathrm{H}$ bending/wagging modes and $\mathrm{C}-\mathrm{C}$ breathing modes (from aromatic intermediates); blue, $\mathrm{C}-\mathrm{H}_{3}$ bending/umbrella modes (aliphatic); orange, $\mathrm{C}-\mathrm{H}$ stretch in $\mathrm{CH}_{3}$ (aliphatic); green, $\mathrm{C}-\mathrm{H}$ stretching modes (aromatic). (B) DFT-computed HREEL spectra for the $\mathrm{C}_{2} \mathrm{H}_{3}$ (ethylidyne), $\mathrm{C}_{4} \mathrm{H}_{4}$ (metallocycle), and $\mathrm{C}_{6} \mathrm{H}_{6}$ (benzene) molecules adsorbed on $\mathrm{Rh}(111)$ based on the optimized geometries found in SI7. For comparison, the colored bars are defined as for (A). (C) Experimental integrated signals for the various $\mathrm{C}-\mathrm{H}$ and $\mathrm{C}-\mathrm{C}$ vibrational frequencies from the HREEL spectra in (A) as a function of annealing temperature. The trace colors correspond to the vibrational signals highlighted by the colored bars in (A).

previously reported ${ }^{27}$ from which we infer that upon adsorption at $300 \mathrm{~K}$ the initial $\mathrm{C}-\mathrm{H}$ bond cleavage induces a $\mathrm{C}-\mathrm{C}$ bond order change to 1 , and results in an upright adsorption geometry (see Figure 1, transformation at $300 \mathrm{~K}$ ). This assignment is further confirmed by the excellent agreement between the corresponding experimental HREEL spectrum (300 $\mathrm{K}$ in Figure $3 \mathrm{a}$ ) and the simulated one, computed from the DFT-optimized geometry of ethylidyne adsorbed on the $\mathrm{Rh}(111)$ surface (Figure 3b, SI3, and SI7).

Thermal annealing of the ethylidyne/ $\mathrm{Rh}(111)$ system to $370 \mathrm{~K}$ results in disordered arrangement of protrusions exhibiting various lateral dimensions and heights (Figure $2 \mathrm{~b}$ ). Close inspection reveals essentially the presence of three distinct species classified by their apparent height. The smaller and most abundant protrusions $(40 \mathrm{pm})$ and the intermediate height protrusions $(100 \mathrm{pm})$ are assigned to adsorbed hydrogen atoms and to $\mathrm{C}_{6}$ rings, respectively, on the basis of independent STM measurements, whereby molecular hydrogen and benzene are exposed to the $\mathrm{Rh}$ surface at room temperature (see SI5). The third species, which has the largest apparent height $(140 \mathrm{pm})$ is identified as a $\mathrm{C}_{4}$ unit (see below).

The sequential transformation of $\mathrm{C}_{2}$ species (ethylidyne) into $\mathrm{C}_{6}$ intermediates points to a surface-assisted $2+2+2$ cyclization mechanism (cyclotrimerization). Indeed, $\mathrm{Rh}$ (as well as other transition metals ${ }^{29,30}$ ) is known to catalyze the formation of benzene. The $\mathrm{C}_{6}$ species is found to coexist with a $\mathrm{C}_{4}$ one (see Figure $2 \mathrm{~b}(370 \mathrm{~K})$ and refs 30 and 31$) ; \mathrm{C}_{4}$ is a metallocycle formed in $2+2$ addition of ethylidyne, known as a reactive intermediate in organometallic catalysis, for example, cyclotrimerization of ethyne. ${ }^{32}$ We note here that the $\mathrm{H}_{2}$ TDS (Figure $2 \mathrm{a}$ ) indicates a $\mathrm{C} / \mathrm{H}$ ratio near $1: 1$ at $370 \mathrm{~K}$. It is likely, however, that the intermediates are not perfectly stoichiometric because saturated benzene is known to be unreactive toward the formation of further surface-bound intermediates. ${ }^{33}$ )

Experimental evidence for the involvement of $\mathrm{C}_{6}$ and $\mathrm{C}_{4}$ species in the temperature range of $370-420 \mathrm{~K}$ is confirmed by HREELS. The full set of observed vibrational modes in the HREEL spectrum for $420 \mathrm{~K}$ (Figure 3a) is only appropriately described in our DFT-based simulations (Figure $3 b$ ) when 


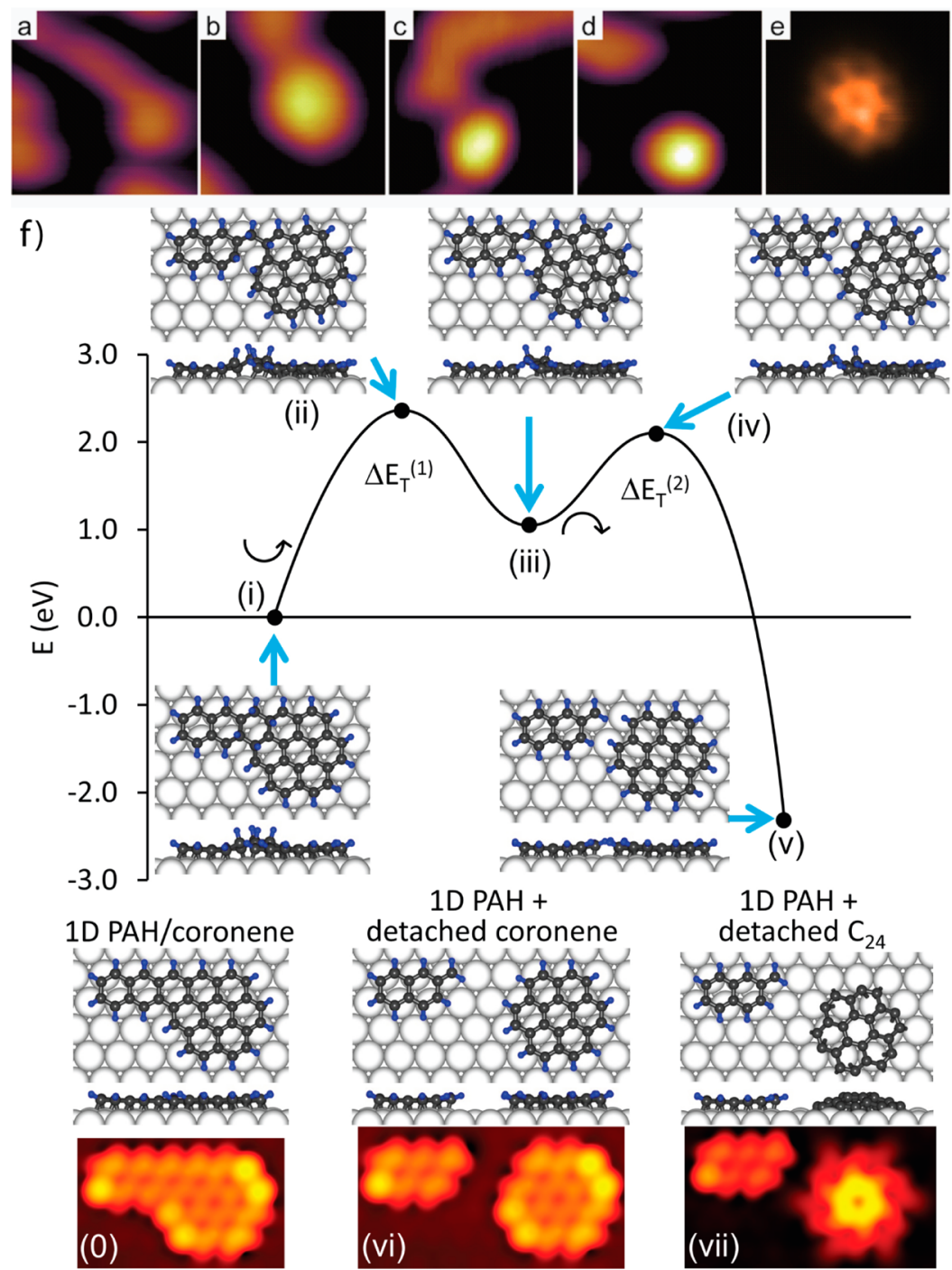

Figure 4. Size-selective PAH fragmentation into coronene-like clusters. (a-e) Selected STM images following a $570 \mathrm{~K}$ annealing of the ethene/ $\mathrm{Rh}$ (111) system. (a) 2D extension of a PAH chain-end, forming a coronene-type termination; (b) Chain-end dehydrogenation yielding a coronenelike carbon cluster termination; (c) Detachment of the terminal coronene-like cluster; and (d) An isolated coronene-like species. Image parameters: $3 \times 3 \mathrm{~nm}^{2}, 0.1$ to $1.1 \mathrm{~V}, 0.1 \mathrm{nA}$. (e) Constant height image acquired on a coronene-like cluster. Image parameters: $2 \times 2 \mathrm{~nm}^{2},-0.1 \mathrm{~V}, 0.05 \mathrm{nA}$. (f) First-principles DFT simulation of the coronene detachment reaction pathway; this simulation considers only enthalpic effects (for considerations of the entropic factor see SI2). The evolution of the detachment process, which entails pivotal in-plane rotations of the detaching 24-carbon-atom cluster, is depicted as a succession of steps: (0)-(vii), with (i) - (v) plotted along the reaction coordinate, and displaying a top and side view of the system for each step. The process starts with configuration (0) depicting an adsorbed coronene attached to the end of the PAH chain; for an essentially indistinguishable simulated STM image obtained with the inclusion of van der Waals (vdW) interactions in the simulations, see SI9. In (i) partial hydrogenation $(4 \mathrm{H})$ of the coronene/PAH junction results in local elevation of the junction carbon atoms. Calculated activation energy barriers, $\Delta E_{\mathrm{T}}(\mathrm{i})$, are indicated, with their values: $2.36 \mathrm{eV}(\mathrm{i}=1)$ and $1.04 \mathrm{eV}(\mathrm{i}=2)$ compatible with the experimental elevated temperature. The corresponding values with the inclusion of vdW interactions in the simulations (see SI9) are 2.39 and $1.00 \mathrm{eV}$, respectively. The curved arrows on the reaction path indicate the sense of pivotal rotation of the detaching 24-carbon-atom cluster (anticlockwise pivotal rotation for the first activation barrier, and clockwise rotation for the second barrier). The process ends with formation of a detached coronene cluster ( $\mathrm{v}$ and vi), which upon dehydrogenation yields an adsorbed carbon cluster. The corresponding simulated STM images illustrate the increasing apparent height in the middle of the dome-shaped detached carbon fragment (compare with e); for a detailed discussion and alternative pathways see SI9.

considering the coexistence of both $\mathrm{C}_{4}$ and $\mathrm{C}_{6}$ species adsorbed on the $\mathrm{Rh}(111)$ surface. Of particular significance is the extended vibrational structure observed upon annealing to $420 \mathrm{~K}$ between 250 and $600 \mathrm{~cm}^{-1}$ (gray-shaded areas in Figures $3 a$ and $3 b$ ) indicative of $\mathrm{Rh}-\mathrm{C}$ stretch frequencies of small planar cyclic or inclined metallocyclic hydrocarbons that develop during the first growth steps. It has to be noted that this characteristic vibrational structure between 250 and 600 $\mathrm{cm}^{-1}$ can only be reproduced by superposition of the corresponding $\mathrm{C}_{4}$ and $\mathrm{C}_{6}$ calculated bands. Hence, the judicious combination of microscopy, spectroscopy, and computational methods concur to support a cyclo-additive mechanism for the first stage of $\mathrm{C}_{2}$ coupling on $\mathrm{Rh}(111)$, yielding $\mathrm{C}_{4}$ and $\mathrm{C}_{6}$ species.

Formation of Segmented 1D Polyaromatic Hydrocarbon Narrow Ribbons. Following a further annealing of the ethene $/ \mathrm{Rh}(111)$ system to $470 \mathrm{~K}$, STM topographies show a transformation into chains of linear segments oriented along

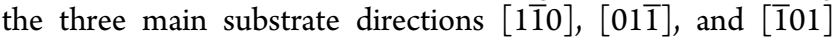
(Figure 2b) with an apparent height of 100 pm. We identify 
these structures as one-dimensional linear polyaromatic hydrocarbons (1D-PAHs, see Figure 1, transformation at $470 \mathrm{~K}$ ) based on several observations (details in SI5): (1) The chains exhibit a longitudinal corrugation periodicity of $2.5 \AA$, in agreement with edge-connected $\mathrm{C}_{6}$ rings. (2) The lateral width of the chains matches that of benzene molecules and is smaller than that of coronene molecules. In addition, the 1D-PAHs, benzene, and coronene have all similar apparent electronic heights of $100 \mathrm{pm}$. (3) STM images of tetracene molecules (as a model for 1D-PAHs with a fixed length of four carbon rings) on $\mathrm{Rh}(111)$ show a remarkable resemblance to the chains. (4) Taking into account the hydrogen balance from TDS (Figure 2a) and the fact that no carbon is lost to the gas phase $^{27}$ nor into the bulk of the $\mathrm{Rh}$ support, ${ }^{21}$ a $\mathrm{C} / \mathrm{H}$ ratio of roughly 2:1 can be deduced for these intermediate species. This stoichiometry rules out all PAHs other than those with the width of a single $\mathrm{C}_{6}$ ring. The corresponding $470 \mathrm{~K}$ HREEL spectrum further confirms the presence of exclusively polyaromatic adsorbates on the $\mathrm{Rh}(111)$ surface, see SI3. Our 1D-PAH results are comparable in all aspects to the constant-current STM images of graphene nanoribbons (GNRs) observed in ref 34 (see in particular the constantcurrent STM image of a kinked narrow GNR in Figure 4a of ref 34; strong coupling between the carbon nanostructures and the $\mathrm{Rh}(111)$, compared to the weak interaction on $\mathrm{Au}(111)$, prevents us from obtaining higher resolution $\mathrm{d} I / \mathrm{d} V$ maps for the adsorbed carbon structures occurring in our study).

Our time-resolved STM measurements, allowing monitoring of processes occurring under continuous exposure to ethene while maintaining the $\mathrm{Rh}$ surface at $455 \mathrm{~K}$ (see SI6 and STM Video 1), confirm the formation of segmented 1D-PAH chains. Moreover, we find that the 1D-PAH growth proceeds continuously at the chain ends with the formation of kinks (see below) but without branching. This supports a growth mechanism where the formation of the 1D-PAHs observed at $470 \mathrm{~K}$ results from sequential addition of $\mathrm{C}_{4}$ units to benzenelike $\mathrm{C}_{6}$ chain seeds (see Figure 1, transformations at $470 \mathrm{~K}$ ). Although the 1D-PAH growth is preferentially linear, inspection of the STM records (Figure $2 b$, see in particular frames at $T=470$ and $520 \mathrm{~K}$ ) reveal $120^{\circ}$ kinks at the ends of $\leq 3 \mathrm{~nm}$ long linear segments (equivalent to 12 benzene rings) oriented along the three main substrate directions $[1 \overline{1} 0],[01 \overline{1}]$, and $[\overline{101}$. This upper limit for the straight segment lengths matches the periodicity of the Moire coincidence lattice resulting from the adsorption of a two-dimensional (2D) graphene monolayer on $\mathrm{Rh}(111) .{ }^{35}$ The lattice mismatch between the substrate $(2.69 \AA)$ and graphene $(2.5 \AA)$ leads to a superstructure (clearly visible on the graphene islands shown in Figure $2 \mathrm{~b}$ at $970 \mathrm{~K}$ ) whereby 12 graphene rings accommodate on $11 \mathrm{Rh}$ atoms (along the high symmetry directions of the substrate), giving rise to an $\sim 3 \mathrm{~nm}$ periodicity of the adsorbed graphene superstructure. The same tensile stress argument is likely to apply to the 1D-PAHs as it becomes energetically preferable to introduce a $120^{\circ}$ kink in order to maintain the chain growth. This is supported by DFT calculations performed for the 1D-PAH shown in Figure 2c (see SI7) with a 12 benzene ring straight segment of $3 \mathrm{~nm}$ in length (between the end edges of the furthest rings) and $2.88 \AA$ in width (between the furthest carbon atoms in the phenyl rings). The height distribution of the carbon atoms along the linear segments is, however, rather weakly modulated (sharp peak at $2.3 \pm 0.1 \AA$ ) in comparison to the height distribution in the Moire pattern of graphene on $\mathrm{Rh}(111)$ (wide variation over the range of
2-4 $\AA^{35}$ ). The highest carbon atoms with respect to the underlying metal surface are found at the edges and in the middle of the linear segment, reflecting a maximal incommensurability (see SI7). The charge transfer from the metal surface to the carbon atoms and the nature of the electronic hybridization are also weakly modulated in a manner that is correlated with the height variations. The bonding is of $\mathrm{sp}^{2}$ character where the chain is high above the surface, whereas it gains some $\mathrm{sp}^{3}$ character when the carbon atoms are closer to the metal surface, reflecting stronger binding with a consequent rehybridization $\left(\mathrm{sp}^{2} \rightarrow \mathrm{sp}^{3}\right)$.

With the identification of ethylidyne at $300 \mathrm{~K}$ and 1D-PAH intermediates at $450 \mathrm{~K}$, the first $\mathrm{H}_{2}$ desorption regime (see Figure $2 \mathrm{a}$ ) is associated with the dehydrogenation of ethene and condensation of aliphatic hydrocarbons into 1D-PAH. The second desorption regime at elevated temperatures (450$700 \mathrm{~K}$ ) is governed by the thermal evolution of $1 \mathrm{D}-\mathrm{PAHs}$ into extended graphene following a $1 \mathrm{D}$ to $2 \mathrm{D}$ structural transition. After prolonged growth at $450 \mathrm{~K}$, the evolved structure is that of mutually avoiding 1D-PAHs (see SI6), indicating that further condensation into 2D-PAH intermediates is inhibited by steric repulsion, and requiring higher temperatures for continuation of the process. The existence of two different growth regimes and their respective interpretation is confirmed by our HREELS investigations. Records of the integrated signals for the various $\mathrm{C}-\mathrm{H}$ vibrational frequencies from HREEL spectra, acquired after successive annealings, summarize the spectroscopic findings, as shown in Figure $3 \mathrm{c}$. From this, we conclude that at $300 \mathrm{~K}$ the predominant frequencies relate solely to aliphatic carbons, as expected. Their corresponding intensities are seen to decrease with temperature, concomitant to the emergence of aromatic carbon fingerprints. Above $420 \mathrm{~K}$, the aliphatic contributions vanish while the aromatic ones are maximized. With further annealing, the aromatic intensities decrease almost monotonically over a wide temperature range (up to $700 \mathrm{~K}$ ), as a consequence of the slow, featureless dehydrogenation ( $\mathrm{C}-\mathrm{H}$ cleavage) observed in the second regime of the TDS data. There remains, however, the question as to how the $1 \mathrm{D}$ to $2 \mathrm{D}$ structural transition proceeds at high temperatures.

Kinking and 1D $\rightarrow$ 2D Dimensionality Crossover. Further kinking of the 1D-PAHs and their consequent broader appearance occurs after annealing at $520 \mathrm{~K}$ (Figure 2b) with the resulting zigzags aligned with the three main symmetry directions of the substrate. While the chain density remains constant upon annealing from 470 to $520 \mathrm{~K}$ (ruling out coalescence), an apparent shortening of the 1D-PAHs by $25 \%$ without change in the apparent height is consistent with the introduction of kinks. This intramolecular reshaping is a consequence of simple thermodynamic arguments (Clar's rule) and can occur by rearrangement of a single, weak, edgeconnecting $\mathrm{C}-\mathrm{C}$ bond while keeping the molecular backbone intact (see SI8). Annealing to $570 \mathrm{~K}$ drives a further shortening of the 1D-PAHs (Figure 2b). In parallel, several chain ends become clearly broader, indicative of the onset of structural reshaping into the second dimension. The increased propensity of kinking can bring the chain ends to curl up (see Figure 1, transformations at 520 and $570 \mathrm{~K}$ ), leading to close proximity of adjoining $\mathrm{C}-\mathrm{H}$ bonds and allowing for $\mathrm{C}-\mathrm{C}$ bond formation at these temperatures; recall the activated formation of graphene nanoribbon on $\mathrm{Au}$ substrates at around $670 \mathrm{~K}^{13}$ Inspection of Figure $2 \mathrm{~b}$ (at $520 \mathrm{~K}$, and particularly $570 \mathrm{~K}$ ) reveals that the broader $\mathrm{PAH}$ chain ends take two distinct 
appearances: one in which the apparent height remains unchanged with respect to the chain segments $(100 \mathrm{pm})$ and the other exhibiting an apparent height increase to $150 \mathrm{pm}$. In addition, these protruding chain ends have a lateral extension of $1 \mathrm{~nm}$, a width reminiscent of that of the size-selective carbon precursors first identified on $\mathrm{Rh}(111)^{21}$ and later on $\mathrm{Ru}(0001) .^{36}$ Examination of the STM records at $570 \mathrm{~K}$ (Figure 2b) confirms that the density of PAH chains decreases at the expense of smaller and detached particles of quasicircular shape, exhibiting a diameter of $1 \mathrm{~nm}$ and a height of up to $150 \mathrm{pm}$, a carbon precursor species with a varying hydrogen content (see below). We note here the ambiguous structural assignment of the carbon precursors: following the work of ref 21 , several reports have confounded the identity of the sizeselective carbon precursors on the $\mathrm{Rh}(111)$ on grounds of $\mathrm{DFT}$ calculations, ${ }^{22,23}$ and to-date no experimental validation has yet emerged. Our present microscopy data precludes further identification. Nevertheless, a lateral extension of $1 \mathrm{~nm}$ suggests a polyaromatic precursor of coronene-type dimensions.

On the basis of these microscopic observations, the following mechanism for the formation of graphene emerges: under appropriate thermal conditions, the 1D-PAH chains-ends undergo $2 \mathrm{D}$-restructuring by a combination of kink formation and curling-up. Coronene-type terminations become apparent in the STM topographs (Figure 4a). Continued 2D-PAH growth beyond this critical size entails detachment of coronenetype species with varying hydrogen content. To substantiate the detachment process, we performed DFT calculations assuming a coronene structure for the carbon precursor (mindful of its contentious structural identification). The results of our DFT calculations reveal that the detachment process may be induced by hydrogen released during the 2D-restructuring. Concomitantly, peripheral dehydrogenation of the detaching species leads to carbon-metal hybridization with the underlying $\mathrm{Rh}$ substrate, forcing the detaching units to adopt a dome-shaped configuration (hence the increased apparent height, Figure 4b). ${ }^{21,37}$ Dehydrogenation may be completed before or after the detachment of the coronene-like unit, resulting in the STM images in Figure $4 \mathrm{c}$,d. These detached, size-selective entities on the $\mathrm{Rh}(111)$ surface that act as precursors to the further growth of graphene ${ }^{21}$ can be identified by constant-height STM that seem to possibly resolve $\mathrm{C}_{6}$ rings (Figure $4 \mathrm{e}$ ). The dehydrogenation processes associated with the above restructuring and disjoining processes of the 1D-PAHs lead to gentle release of $\mathrm{H}_{2}$ to the gas phase, correlating with the TDS findings in the range of 450 to $700 \mathrm{~K}$ (Figure $2 \mathrm{a}$ ).

The slow dehydrogenation process and the concomitant increase in lateral extension of the aromatic backbone are clearly evidenced in the HREEL spectra (Figure 3a) for temperatures above $450 \mathrm{~K}$. Indeed, the intensity of the aromatic $\mathrm{C}-\mathrm{H}$ stretch peak at $3000 \mathrm{~cm}^{-1}$ slowly decreases with temperature and vanishes at $700 \mathrm{~K}$ (as expected from our hydrogen TDS data, Figure 2a). In a concerted manner, one also observes the decrease in intensity of the $750 \mathrm{~cm}^{-1}$ peak, which arises from $\mathrm{C}-\mathrm{H}$ out-of-plane wagging and $\mathrm{C}-\mathrm{C}$ breathing modes (aromatic). While we can rationally infer that the $\mathrm{C}-\mathrm{H}$ wag component must also vanish by $700 \mathrm{~K}$ with the loss of hydrogen, the $\mathrm{C}-\mathrm{C}$ breathing component must remain present at even higher temperatures, when graphene flakes emerge. In addition, the $\mathrm{C}-\mathrm{C}$ breathing modes have mainly dipolar contributions parallel to the surface, and HREELS (operated in specular geometry) is sensitive only to perpendicular dipolar contributions. Hence, the growth of graphene into ever larger flakes is accompanied by a decrease in HREELS intensity for the $\mathrm{C}-\mathrm{C}$ breathing modes and a shift to lower frequency. Interestingly, the $\mathrm{C}-\mathrm{C}$ breathing modes are also seen to broaden with temperature. This is to be expected due to it averaging over the many $\mathrm{Rh}-\mathrm{C}$ bonding configurations imparted by the lattice mismatch between graphene and the Rh substrate, as discussed previously.

Microscopic Formation Mechanism of the SelfEvolved Precursors and Graphene Growth. The pathway of the coronene-like detachment process, obtained from steered first-principles simulations, is displayed in Figure 4f (see SI9). The starting configuration (0) corresponds to a curled-up (surface-lifted) PAH chain-end configuration. The detachment of this terminal unit requires activation (weakening) of the $\mathrm{C}-\mathrm{C}$ bonds bridging the $\mathrm{PAH}$ and the attached coronene through their partial hydrogenation that thus acquire $\mathrm{sp}^{3}$ character. This process is akin to a hydrogenolysis-type mechanism defined as a chemical reaction whereby a carbon-carbon undergoes "lysis" by hydrogen. ${ }^{38}$ The detachment process of the carbon cluster from the thickened PAH chain-ends was found to occur at about $570 \mathrm{~K}$ (see Figure $2 \mathrm{~b}$ $(570 \mathrm{~K}))$. At this temperature, hydrogen evolution on the surface is still observed, as evidenced by the measured hydrogen desorption spectrum displayed in Figure $2 \mathrm{a}$; in fact, the hydrogen desorption signal vanishes only at temperatures well above that of the detachment process, that is, above $700 \mathrm{~K}$ (see Figure 2a). Therefore, it is most reasonable to expect that hydrogen atoms in sufficient abundance are available for capture and local hydrogenation/activation of the bonds linking the carbon cluster to the polyaromatic hydrocarbon chain as conjectured by the microscopic detachment mechanism simulated and described in Figure $4 \mathrm{f}$.

Thereafter, the detachment mechanism depicted in Figure $4 \mathrm{f}$ involves successive breaking of these $\mathrm{C}-\mathrm{C}$ bonds. The evolution of the detachment process entails pivotal in-plane rotations of the detaching 24-carbon-atom cluster (see Figure 4f, and Figures S26 and S27 where alternative detachment pathways are shown with overall characteristics similar to the pathway displayed in Figure 4f). Such pivotal cluster rotations have been discovered earlier ${ }^{39}$ to underlie cluster diffusional (and superdiffusional, that is, Lévy flights) motion ${ }^{40-42}$ on incommensurate solid surfaces (as is the case for the interface between the size-selective cluster and the $\mathrm{Rh}(111)$ surface mesh). After detachment, the PAH chain residues are likely to diffuse, merge, and perpetuate the production of size-selective clusters. Increased annealing leads to thermal diffusion (involving the aforementioned in-plane cluster rotations) of the precursors, ${ }^{21}$ which coalesce into extended graphene.

The microscopic pathways described here operate over a wide temperature range and allow for a slow release of the remaining $25 \%$ hydrogen to the gas phase, compatible with our TDS data (Figure 2a). The surface-catalyzed thermally activated chemical transformation of adsorbed ethene into graphene (Figure 1), which we found to proceed via coupling reactions that lead to formation of segmented 1D-PAH chains with a subsequent dimensionality crossover $(1 \mathrm{D} \rightarrow 2 \mathrm{D})$ and restructuring, resulting in eventual detachment of self-evolved precursors (Figure 4), culminates at 770 to $990 \mathrm{~K}$ with diffusional self-assembly and condensation of the precursors to form extended graphene monolayer patches with a high degree of structural perfection, as displayed in Figure $2 \mathrm{~b}$. We note here that the maximum graphene coverage attainable under our experimental conditions is limited by the number of ethylene 
molecules deposited at $300 \mathrm{~K}$ to $21 \%$ of the total $\mathrm{Rh}$ surface, because no carbon atoms are lost to the gas phase nor to the bulk of the $\mathrm{Rh}$ support during our annealing experiments. ${ }^{21}$

\section{CONCLUSIONS}

When employing an appropriate programmed sequence of temperature windows, thereby regulating and controlling the subtle balance between kinetics and thermodynamics, the surface-catalyzed chemical transformation of adsorbed ethene into graphene (Figure 1), studied here on single crystal $\mathrm{Rh}(111)$ under ultrahigh vacuum conditions, can be directed toward the controlled formation of polyaryl intermediates (and products) with precise morphologies: 1D-PAH chains, sizeselective 2D-PAH clusters, and extended single-layer graphene. The observed dimensionality crossover $(1 \mathrm{D} \rightarrow 2 \mathrm{D})$, resulting from polyaryl dynamical restructuring, ushers the emergence of a self-evolved precursor resulting from detachment of a coronene-type end-chain-unit (Figure 4) and culminates with rate-limiting diffusional self-assembly and condensation into extended graphene monolayer patches with a high degree of structural perfection (Figure $2 \mathrm{~b}$ ). These results suggest future explorations of the paradigm of dynamically self-evolved precursors in surface-catalyzed reactions through judicial selection of substrate and adsorbate materials, surface processing, and systematic programmed variation of the ambient reaction conditions.

\section{EXPERIMENTAL METHODS}

Methodology for the Low-Temperature STM Measurements. Experiments were performed in an ultrahigh vacuum (UHV) surface analysis system with a base pressure below $1 \times 10^{-10} \mathrm{mbar}$, consisting of a preparation chamber allowing for standard sample preparation and characterization by Auger electron spectroscopy (AES), and a microscope chamber housing a CreaTec low-temperature STM. The $\mathrm{Rh}(111)$ crystal was cleaned by repeated cycles of $\mathrm{Ar}^{+}$ sputtering and annealing in oxygen $\left(3 \times 10^{-7} \mathrm{mbar}\right)$ at 1000 $\mathrm{K}$ and finalized by flash-annealing in vacuum at $1000 \mathrm{~K}$. STM acquisition was performed at liquid nitrogen $\left(\mathrm{LN}_{2}\right)$ temperature using the constant-current mode and homemade $\mathrm{W}$ tips. The cleanliness of the surface was monitored by STM and AES. Research-grade ethene, $\mathrm{C}_{2} \mathrm{H}_{4}$ (99.995\% purity), was exposed to the $\mathrm{Rh}$ surface up to saturation levels (approximately 5 Langmuir, where $1 \mathrm{~L}=1.33 \times 10^{-6} \mathrm{mbar} / \mathrm{s}$ ) at room temperature, followed by a series of sequential annealings up to $973 \mathrm{~K}$ in UHV. A typical annealing consists of heating the sample to the desired temperature for $30 \mathrm{~s}$, after which it is quenched down to $\mathrm{LN}_{2}$ temperature for STM data acquisition.

Methodology for the Time-Resolved STM Measurements. Experiments were performed in an ultrahigh vacuum (UHV) surface analysis system, with a base pressure below $2 \times$ $10^{-10}$ mbar, consisting of a preparation chamber allowing for standard sample preparation and characterization by AES and quadrupole mass spectroscopy (QMS), and a microscope chamber housing a Omicron VT-STM/AFM. The Rh(111) crystal was cleaned by repeated cycles of $\mathrm{Ar}^{+}$sputtering and annealing in vacuum at $1250 \mathrm{~K}$. STM acquisition was performed between 300 and $825 \mathrm{~K}$ using the constant-current mode and homemade $\mathrm{W}$ tips. The sample was resistively heated. For the STM measurements at constant temperature, the heating power was kept constant and no drift correction had to be applied. For the STM measurements at increasing temperature, the heating current was increased slowly, by 0.1 $\mathrm{mA}(\sim 0.6 \mathrm{~mW})$ at fixed time steps of $10 \mathrm{~s}$, leading to temperature increases of $\sim 0.05 \mathrm{~K}$. The induced drift was corrected via lateral feedback. The cleanliness of the surface was monitored by STM, AES, and QMS. Ethene, $\mathrm{C}_{2} \mathrm{H}_{4}(99.5 \%$ purity), was exposed to the $\mathrm{Rh}$ surface. First, when dosing at room temperature, exposures of $27 \mathrm{~L}$ were applied, after which the substrate was annealed to higher temperatures. Second, when dosing at elevated temperatures, ethene was exposed continuously at approximately $5 \times 10^{-9}$ mbar during the STM acquisition.

Methodology for the HREELS Measurements. Within a dedicated experimental system, composed of a preparation chamber with base pressure below $5 \times 10^{-10} \mathrm{mbar}$ and an analysis chamber with base pressure below $1 \times 10^{-10} \mathrm{mbar}$, HREELS measurements were carried out in the specular direction $\left(\theta_{\mathrm{i}}=\theta_{\mathrm{f}}=45^{\circ}\right)$ with a primary beam energy of $4 \mathrm{eV}$ and a typical elastic peak resolution of about $50 \mathrm{~cm}^{-1}(6.2 \mathrm{meV}$ fwhm). A maximum likelihood-based resolution enhancement $\operatorname{method}^{43,44}$ was used to recover the spectra from the instrumental broadening, leading to an improved resolution of about $40 \mathrm{~cm}^{-1}$ fwhm. Spectra were normalized to the intensity of the elastic peak. Ethene $\left(\mathrm{C}_{2} \mathrm{H}_{4}, 99.995 \%\right.$ purity $)$ was dosed at room temperature $(300 \mathrm{~K})$ for an exposure of 2,5 , and $10 \mathrm{~L}$, enough to ensure saturation coverage. The substrate was then flash-annealed to progressively higher temperatures and HREEL spectra collected after allowing the sample to cool to room temperature. Similar behavior is observed following each of the exposures; the $5 \mathrm{~L}$ experiment is discussed as a typical example of the spectral evolution seen upon sequential flash annealing.

\section{COMPUTATIONAL DETAILS}

Density Functional Calculations. Calculations were performed using the VASP-DFT package with a plane-wave basis (kinetic-energy cutoff of $400 \mathrm{eV}$ ), PAW pseudopotentials, ${ }^{45}$ and the PBE generalized gradient approximation (GGA) for the exchange-correlation potential. ${ }^{46}$ Because the unit cell used here is rather large, we have used in most of our calculations a single $k$-point sampling ( $\Gamma$-point) of the surface Brillouin zone (SBZ); we have checked that the results remain very similar by employing a $(3 \times 3 \times 1)$ SBZ sampling. In optimization of the various structures, convergence was achieved when the calculated forces were smaller than 0.005 $\mathrm{eV} / \AA$. In the simulations, the $\mathrm{Rh}(111)$ substrate surface consisted of three layers with the optimized $\mathrm{Rh}$ lattice parameter $a=2.718 \AA$, agreeing with the experimental values ( $a=2.69 \AA$ ). In structural relaxations, the bottom layer of the substrate slab was held fixed. In simulations of the configuration of long linear 1D-PAH chain with two kinks (see Figure 2b), the supercell had a $(18 \times 10)$ lateral periodicity of the threelayer $\mathrm{Rh}(111)$ slab, and a vacuum region that was taken large enough to ensure no interaction between periodic replicas; for the bare $\mathrm{Rh}(111)$ slab, the vacuum region was taken as $20.7 \AA$. In simulations of configuration, the 24 carbon atom cluster (or coronene) configuration with a 1D-PAH tail (see Figure 4 ), the supercell had an $(10 \times 8)$ lateral periodicity of the three-layer $\mathrm{Rh}(111)$ slab, and in simulation of the $\mathrm{C}_{4} \mathrm{H}_{4}$ and $\mathrm{C}_{6} \mathrm{H}_{6}$ intermediates (see Figure $2 \mathrm{~d}$ ) the supercell had an $(12 \times 6)$ lateral periodicity.

Simulations of Reaction Pathways. In simulations of the reaction pathways (in particular the detachment of the 24carbon atom cluster from the PAH tail (see Figure 4f)), we 
have used steered first-principles simulations, where a certain reaction coordinate (or reaction coordinates, which may consist of certain interatomic distances, angles, and/or combinations thereof) is varied in a controlled way, whereas all other nuclear degrees of freedom are allowed to fully relax after each increment of the reaction coordinate. The relaxation process is performed by using an algorithm based on a conjugated gradient method with the total energy evaluated at each step of the relaxation process quantum mechanically using the density functional theory (DFT) described above. In this way, the activation energy barriers are determined; once the top of an activation barrier is reached, the steering of the reaction is stopped and the system's nuclear coordinates are allowed to evolve freely on the concomitantly first-principles-calculated potential energy surface until the nearest potential energy minimum is reached. If the minimum reached corresponds to the desired reaction products, the simulation is stopped. On the other hand, if the potential energy minimum reached is a local one, steering of the reaction resumes along a judiciously chosen reaction coordinate, and the next activation barrier is determined. Often several alternative reaction coordinates are employed and the one yielding the lowest activation energy barrier is selected. These calculations yield results that are the same as, or close to, those obtained by other methods (e.g., the nudged elastic band and variants thereof; see discussion in ref 47).

In the present study, we restricted ourselves to consideration of the enthalpy contribution to the reaction profile. Free-energy reaction profiles (that is, including entropic effects) can be obtained through a sequence of canonical (constant-temperature) first-principle (Born-Oppenheimer) molecular dynamics (FPBOMD) simulations while constraining the reaction coordinate to prescribed values (see Results and Discussion above), and calculation of the reversible work done in taking the system along the reaction path between the reactant and product states. However, the exceedingly large computational demands of such first-principle calculations limits the methods to systems much smaller than the one investigated here. Furthermore, because the reaction that we discuss here involves breaking of a rather strong bond (the $\mathrm{C}-\mathrm{C}$ bond) it is reasonable to assume that potential energy (enthalpy) effects will dominate the reaction profile and that the inclusion of entropic ones (mainly vibrational entropy of the adsorbed complex) will not modify our results in an essential way.

van der Waals (vdW) Interactions. Inclusion of $\mathrm{vdW}$ interactions ${ }^{48}$ resulted in changes in the total energies of the systems being investigated. However, structural relaxations with and without the inclusion of $\mathrm{vdW}$ interactions yielded essentially the same optimal structures. Moreover, the energy barriers determined from our simulations show very small dependence on the vdW interactions. See further details in the Supporting Information.

Calculations of STM Images. STM images were simulated by using the Tersoff-Hamann approximation ${ }^{49}$ in which the tunneling current is considered to be proportional to the integrated local density of states within a given energy window determined by the applied bias on the sample; we have used an energy window of $1 \mathrm{eV}$ below the Fermi energy, that is, $E_{\mathrm{F}}-1$ $\mathrm{eV}<E<E_{\mathrm{F}}$, corresponding to a bias voltage of $-1 \mathrm{~V}$. These STM images are topographic images (that is, the color variations correspond to different heights) because the images correspond to those recorded in constant current scans.
Simulations of HREEL Spectra. $\mathrm{C}_{2} \mathrm{H}_{3}$ (ethylidyne), $\mathrm{C}_{4} \mathrm{H}_{4}$ and $\mathrm{C}_{6} \mathrm{H}_{6}$ isolated species (all on $4 \times 4$ cell sizes) were optimized using a higher convergence criterion to obtain the simulated spectra. In particular, the convergence criterion for the electronic self-consistent cycle was fixed at $10^{-6} \mathrm{eV} / \AA$ and the forces on all ions were required to be smaller than 0.015 $\mathrm{eV} / \AA$. The Born effective charges (BEC) matrix for each system were obtained employing density functional perturbation theory inherent in VASP. Intensities were calculated using a similar procedure as found in reference ${ }^{50}$ where the BEC tensor was projected to the perpendicular direction with respect to the surface. The intensity-weighted harmonic eigenfrequencies were broadened by applying a Gaussian function with a fwhm of $40 \mathrm{~cm}^{-1}$, corresponding to the resolution of the experimental HREELS setup. In addition, the spectra were corrected for the spectrometer transfer function, as described in ref 51 .

\section{ASSOCIATED CONTENT}

\section{S Supporting Information}

The Supporting Information is available free of charge on the ACS Publications website at DOI: 10.1021/acs.jpcc.7b01999.

Methodology of the experiments; methodology of the DFT calculations; HREEL spectroscopy studies; highresolution STM images of the ethene/ $\mathrm{Rh}(111)$ system after deposition and after annealing to successively higher temperatures; the initial stages of ethene condensation on $\mathrm{Rh}(111)$ as observed by STM; time-resolved STM data on the 1D-PAH chain growth at $455 \mathrm{~K}$; the intermediates identified during ethene condensation on $\mathrm{Rh}(111)$ as modeled by DFT calculations; 1D-PAH intramolecular reshaping; DFT calculation results on the formation of coronene-like clusters-mechanistic details and alternative pathways (PDF)

STM time series of the ethene decomposition at $455 \mathrm{~K}$, dynamics of 1D-PAH growth (AVI)

\section{AUTHOR INFORMATION}

\section{Corresponding Authors}

*E-mail: (F.E.) friedrich.esch@tum.de.

*E-mail: (U.L.) uzi.landman@physics.gatech.edu.

*E-mail: (R.S.) renald.schaub@st-andrews.ac.uk.

ORCID

Michael-John Treanor: 0000-0002-8613-0337

José A. Garrido Torres: 0000-0002-1727-0862

Marco Caffio: 0000-0002-9373-6054

Federico Grillo: 0000-0001-9961-1212

Herbert Früchtl: 0000-0001-6647-4266

Friedrich Esch: 0000-0001-7793-3341

Ueli Heiz: 0000-0002-9403-1486

Uzi Landman: 0000-0002-1586-1554

Renald Schaub: 0000-0002-1712-3336

\section{Author Contributions}

"B.W. and M.K. contributed equally. R.S., F.E., B.W., U.H., and U.L. conceived and designed the study. B.W., M.K., C.J.B., M.J.T., and M.C. prepared specimens, took STM images, and conducted image processing. F.G. and C.J.B. prepared specimens, took HREELS spectra and conducted spectroscopic analysis. B.Y. and U.L. conducted and analyzed DFT calculations. J.A.G.T. and H.F. computed the HREEL spectra from the DFT calculations. All of the authors discussed the 
experimental results. All of the authors contributed to the interpretation of the data. B.W. and M.K. contributed to the initial elaboration of the manuscript; R.S., U.L.. and F.E. wrote the manuscript.

\section{Notes}

The authors declare no competing financial interest.

The research data supporting this publication can be accessed at http://dx.doi.org/10.17630/dcf140b9-ea78-4cd7-a279c8d19e6e42b7.

\section{ACKNOWLEDGMENTS}

We acknowledge Prof. Christopher J. Baddeley, University of St Andrews, for insightful discussions and critical manuscript reviewing. The experimental work at the TU Munich was funded by the Deutsche Forschungsgemeinschaft (DFG, projects ES 349/1-1 and HE 3454/18-1). Financial support by the Friedrich Ebert-Stiftung to M.K. is gratefully acknowledged. The work of B.Y. at the Georgia Institute of Technology was supported by Grant FA9550-14-1-0005 from the U.S. Air Force Office of Scientific Research, and the work of U.L. was supported in part by a Grant FG05-86ER45234 from the Office of Basic Energy Sciences of the U.S. Department of Energy. Calculations were performed at the Georgia Institute of Technology Center for Computational Materials Science. R.S. and N.V.R. acknowledge financial support from the Scottish Funding Council through EaStCHEM and SRDG Grant HR07003. R.S. and H.F. acknowledge EPSRC for the use of the ARCHER U.K. National Supercomputing service, and for the funding of $\mathrm{PhD}$ studentships (JAGT - EP/M506631/1, and MJT - EP/K503162/1).

\section{REFERENCES}

(1) Moulijn, J. A.; van Diepen, A. E.; Kapteijn, F. Catalyst deactivation: Is it predictable? What to do? Appl. Catal., A 2001, $212,3-16$

(2) Forzatti, P.; Lietti, L. Catalyst deactivation. Catal. Today 1999, $52,165-181$

(3) Galloway, R. L. A History of coal mining in Great Britain. In National Archives U.K., 1883; Chapter 5.

(4) Novoselov, K. S.; Geim, A. K.; Morosov, S. V.; Jiang, D.; Zhang, Y.; Dubonos, S. V.; Grigorieva, I. V.; Firsov, A. A. Electric field effect in atomically thin carbon films. Science 2004, 306, 666-669.

(5) Novoselov, K. S.; Falko, V. I.; Colombo, L.; Gellert, P. R.; Schwab, M. G.; Kim, K. A roadmap for graphene. Nature 2012, 490, 192-200.

(6) Tour, J. M. Top-down versus bottom-up fabrication of graphenebased electronics. Chem. Mater. 2014, 26, 163-171.

(7) Luo, B.; Liu, S.; Zhi, L. Chemical approaches toward graphenebased nanomaterials and their applications in energy-related areas. Small 2012, 8, 630-646.

(8) Li, X.; Wang, X.; Zhang, L.; Lee, S.; Dai, H. Chemically derived, ultrasmooth graphene nanoribbon semiconductors. Science 2008, 319, $1229-1232$

(9) Kosynkin, D. V.; Higginbotham, A. L.; Sinitskii, A.; Lomeda, J. R.; Dimiev, A.; Price, B. K.; Tour, J. M. Longitudinal unzipping of carbon nanotubes to form graphene nanoribbons. Nature 2009, 458, 872876.

(10) Haddon, R. C. Graphene - the mother of two-dimensional (2D) materials. Acc. Chem. Res. 2013, 46, 2191-2192.

(11) Vo, T. H.; Shekhirev, M.; Kunkel, D. A.; Morton, M. D.; Berglund, E.; Kong, L.; Wilson, P. M.; Dowben, P. A.; Enders, A.; Sinitskii, A. Large-scale solution synthesis of narrow graphene nanoribbons. Nat. Commun. 2014, 5, 3189.

(12) Colombo, L.; Wallace, R. M.; Ruoff, R. S. Graphene growth and device integration. Proc. IEEE 2013, 101, 1536-1556.
(13) Cai, J. M.; et al. Atomically precise bottom-up fabrication of graphene nanoribbons. Nature 2010, 466, 470-473.

(14) Bae, S.; et al. Roll-to-roll production of 30-in. graphene films for transparent electrodes. Nat. Nanotechnol. 2010, 5, 574-578.

(15) Müllen, K. Evolution of graphene molecules: Structural and functional complexity as driving forces behind nanoscience. ACS Nano 2014, 8, 6531-6541.

(16) Somorjai, G. A.; Marsh, A. L. Active sites and states in the heterogeneous catalysis of carbon-hydrogen bonds. Philos. Trans. $R$. Soc., A 2005, 363, 879-900.

(17) Tetlow, H.; Posthuma de Boer, J.; Ford, I. J.; Vvedensky, D. D.; Coraux, J.; Kantorovich, L. Growth of epitaxial graphene: Theory and experiment. Phys. Rep. 2014, 542, 195-295.

(18) Loginova, E.; Bartelt, N. C.; Feibelman, P. J.; McCarthy, K. F. Factors influencing graphene growth on metal surfaces. New J. Phys. 2009, 11, 063046.

(19) Loginova, E.; Bartelt, N. C.; Feibelman, P. J.; McCarthy, K. F. Evidence for graphene growth by $\mathrm{C}$ cluster attachment. New J. Phys. 2008, 10, 093026.

(20) Tetlow, H.; Posthuma de Boer, J.; Ford, I. J.; Vvedensky, D. D.; Curcio, D.; Omiciuolo, L.; Lizzit, S.; Baraldi, A.; Kantorovich, L. Ethylene decomposition on $\operatorname{Ir}(111)$ : Initial path to graphene formation. Phys. Chem. Chem. Phys. 2016, 18, 27897-27909.

(21) Wang, B.; Ma, X. F.; Caffio, M.; Schaub, R.; Li, W. X. Sizeselective carbon nanoclusters as precursors to the growth of epitaxial graphene. Nano Lett. 2011, 11, 424-430.

(22) Yuan, Q.; Gao, J.; Shu, H.; Zhao, J.; Chen, X.; Ding, F. Magic carbon clusters in the chemical vapor deposition growth of graphene. J. Am. Chem. Soc. 2012, 134, 2970-2975.

(23) Gao, J.; Ding, F. The structure and stability of magic carbon clusters observed in graphene chemical vapor deposition growth on $\mathrm{Ru}(0001)$ and $\mathrm{Rh}(111)$ surfaces. Angew. Chem., Int. Ed. 2014, 53, 14031-14035.

(24) Zangwill, A.; Vvedensky, D. D. Novel growth mechanism of epitaxial graphene on metals. Nano Lett. 2011, 11, 2092-2095.

(25) Günther, S.; Dänhardt, S.; Ehrensperger, M.; Zeller, P.; Schmitt, S.; Wintterlin, J. High-temperature scanning tunneling microscopy study of the ordering transition of an amorphous carbon layer into graphene on ruthenium(0001). ACS Nano 2013, 7, 154-164.

(26) Wander, A.; Van Hove, M. A.; Somorjai, G. A. Moleculeinduced displacive reconstruction in a substrate surface: Ethylene adsorbed on $\mathrm{Rh}(111)$ studied by low-energy-electron-diffraction. Phys. Rev. Lett. 1991, 67, 626-628.

(27) Dubois, L. H.; Castner, D. G.; Somorjai, G. A. The chemisorption of acetylene and ethylene on $\mathrm{Rh}(111)$ - a low-energy electron-diffraction (LEED), high-resolution electron-energy loss (ELS), and thermal-desorption mass-spectrometry (TDS) study. J. Chem. Phys. 1980, 72, 5234-5240.

(28) Basaran, D.; Aleksandrov, H. A.; Chen, Z.-X.; Zhao, Z.-J.; Rösch, N. Decomposition of ethylene on transition metal surfaces $\mathrm{M}(111)$. A comparative DFT study of model reactions for $\mathrm{M}=\mathrm{Pd}, \mathrm{Pt}, \mathrm{Rh}, \mathrm{Ni}$. J. Mol. Catal. A: Chem. 2011, 344, 37-46.

(29) Amer, I.; Bernstein, T.; Eisen, M.; Blum, J.; Vollhardt, K. P. C. Oligomerization of alkynes by the $\mathrm{RhCl}_{3}$-Aliquat 336 catalyst system: Part 1. Formation of benzene derivatives. J. Mol. Catal. 1990, 60, 313321.

(30) Lambert, R. M.; Ormerod, R. M. Heterogeneously catalyzed cyclization reactions of ethyne over single-crystal palladium and palladium catalysts. Mater. Chem. Phys. 1991, 29, 105-115.

(31) Ormerod, R. M.; Lambert, R. M.; Hoffmann, H.; Zaera, F.; Yao, J. M.; Saldin, D. K.; Wang, L. P.; Bennett, D. W.; Tysoe, W. T. NEXAFS identification of a catalytic reaction intermediate: $\mathrm{C}_{4} \mathrm{H}_{4}$ on Pd(111). Surf. Sci. 1993, 295, 277-286.

(32) Rosenthal, U.; Burlakov, V. V.; Bach, M. A.; Beweries, T. Fivemembered metallacycles of titanium and zirconium - attractive compounds for organometallic chemistry and catalysis. Chem. Soc. Rev. 2007, 36, 719-728. 
(33) Ormerod, R. M.; Lambert, R. M. Heterogeneously catalysed cyclotrimerisation of ethyne to benzene over supported palladium catalysts. J. Chem. Soc., Chem. Commun. 1990, 1421-1423.

(34) Kimouche, A.; Ervasti, M. M.; Drost, R.; Halonen, S.; Harju, A.; Joensuu, P. M.; Sainio, J.; Liljeroth, P. Ultra-narrow metallic armchair graphene nanoribbons. Nat. Commun. 2015, 6, 10177.

(35) Wang, B.; Caffio, M.; Bromley, C.; Früchtl, H.; Schaub, R. Coupling epitaxy, chemical bonding, and work function at the local scale in transition metal-supported graphene. ACS Nano 2010, 4, 5773-5782.

(36) Cui, Y.; Fu, Q.; Zhang, H.; Bao, X. Formation of identical-size graphene nanoclusters on $\mathrm{Ru}(0001)$. Chem. Commun. 2011, 47, 14701472.

(37) Lacovig, P.; Pozzo, M.; Alfe, D.; Vilmercati, P.; Baraldi, A.; Lizzit, S. Growth of dome-shaped carbon nanoislands on $\operatorname{Ir}(111)$ : The intermediate between carbidic clusters and quasi-free-standing graphene. Phys. Rev. Lett. 2009, 103, 166101.

(38) Connor, R.; Adkins, H. Hydrogenolysis of oxygenated organic compounds. J. Am. Chem. Soc. 1932, 54, 4678-4690.

(39) Luedtke, W.; Landman, U. Slip diffusion and Lévy flights of an adsorbed gold nanocluster. Phys. Rev. Lett. 1999, 82, 3835-3838.

(40) Bardotti, L.; Jensen, P.; Hoareau, A.; Treilleux, M.; Cabaud, B.; Perez, A.; Cadete Santos Aires, F. Diffusion and aggregation of large antimony and gold clusters deposited on graphite. Surf. Sci. 1996, 367, 276-292.

(41) Bréchignac, C.; Cahuzac, P.; Carlier, F.; Colliex, C.; Leroux, J.; Masson, A.; Yoon, B.; Landman, U. Instability driven fragmentation of nanoscale fractal islands. Phys. Rev. Lett. 2002, 88, 196103.

(42) Yoon, B.; Akulin, V. M.; Cahuzac, P.; Carlier, F.; de Frutos, M.; Masson, A.; Mory, C.; Colliex, C.; Bréchignac, C. Morphology control of the supported islands grown from soft-landed clusters. Surf. Sci. 1999, 443, 76-88.

(43) Frederick, B. G.; Richardson, N. V. Comment on "Ultrahigh resolution electron energy loss spectroscopy”. Phys. Rev. Lett. 1994, 73, 772.

(44) Frederick, B. G.; Nyberg, G. L.; Richardson, N. V. Spectral restoration in HREELS. J. Electron Spectrosc. Relat. Phenom. 1993, 64$65,825-834$.

(45) Kresse, G.; Joubert, D. From ultrasoft pseudopotentials to the projector augmented-wave method. Phys. Rev. B: Condens. Matter Mater. Phys. 1999, 59, 1758-1775.

(46) Perdew, J. P.; Burke, K.; Ernzerhof, M. Generalized gradient approximation made simple. Phys. Rev. Lett. 1996, 77, 3865-3868.

(47) Heiz, U.; Landman, U. In Nanocatalysis; Springer-Verlag: Berlin, 2007; pp 89-90.

(48) Grimme, S. Semiempirical GGA-type density functional constructed with a long-range dispersion correction. J. Comput. Chem. 2006, 27, 1787-1799.

(49) Tersoff, J.; Hamann, D. R. Theory and application for the scanning tunnelling microscope. Phys. Rev. Lett. 1983, 50, 1998-2001. (50) Karhánek, D.; Bučko, T.; Hafner, J. A density-functional study of the adsorption of methane-thiol on the (111) surfaces of the Ni-group metals: II. Vibrational spectroscopy. J. Phys.: Condens. Matter 2010, 22, 265006.

(51) Ibach, H. Electron energy loss spectrometers. In Springer Series in Optical Sciences; Hawkes, P. W., Ed.; Springer-Verlag, 1991; Vol. 63. 